\title{
Éditorial
}

\section{Le chanvre industriel - perspectives et réalités}

lante connue par ses applications dans la pharmacopée chinoise depuis 2727 avant JC, le chanvre suscite depuis une vingtaine d'années un vif regain d'intérêt tant dans le monde industriel que dans la communauté scientifique.

Qu'une revue comme Matériaux et Techniques y consacre un numéro thématique en est une preuve supplémentaire. Essayons donc, dans cette introduction, de faire le point sur les réalités et les perspectives d'usage et de développement, en terminant sur les conditions à remplir pour connaître un déploiement important et durable de la culture et de l'emploi du chanvre industriel.

À date, il a été cultivé, en 2010, en France, presque 12000 ha. Ce qui fait de notre pays le $2^{\mathrm{e}}$ producteur mondial derrière. . . la Chine (bien sûr !) avec quelques 100000 ha. Le total mondial affiche avec 130000 ha une constante progression depuis les années 1990.

Pourquoi ? Trois faits expliquent le phénomène.

Pour une large part, de nouvelles utilisations sont venues relayer les emplois traditionnels de la fibre (papier) et de la chènevotte (litières animales). Nous y reviendrons plus loin.

D'autre part, les législations restrictives se sont allégées et clarifiées (en Europe et au Canada tout particulièrement) donnant ainsi des marges de manœuvre nouvelles aux opérateurs.

Enfin, comme dit plus haut, les chinois ont mis en œuvre des productions importantes pour alimenter l'industrie textile. $^{1}$

\section{Les réalités actuelles de l'industrie chanvrière sont encourageantes}

Quelques exemples le montreront dans les emplois des composants du chanvre industriel (fibre, chènevotte et graine) et en observant le développement des entreprises chanvrières.

Pendant 40 ans, après la $2^{\mathrm{e}}$ guerre mondiale, le chanvre a été cultivé exclusivement pour fabriquer des papiers spéciaux (ultra fins). Puis sont nées de nouvelles applications, telle la laine de chanvre ${ }^{2}$. Cette fibre est maintenant employée en composant de matériaux thermoformables et/ou thermoplastiques.

Plusieurs entreprises se sont positionnées sur ces différents marchés tels Agrochanvre produisant des PVC chargés en chanvre ou Cavac Biomatériaux qui a construit une chaine complète depuis la production de chanvre jusqu'à la fabrication de laine de chanvre.

Remarquable aussi, le très considérable développement de l'emploi de la chènevotte comme matériau servant à fabriquer des bétons de chanvre ou des blocs à maçonner, grâce à l'association Construire en Chanvre $(\mathrm{CenC})^{3}$. Les professionnels qui la constituent ont à leur actif la mise au point de procédés éprouvés, maintenant reconnus et codifiés dans les Règles Professionnelles d'Exécution d'Ouvrages en Béton de Chanvre. Ces règles s'appuient sur deux piliers : le bon fonctionnement des matériaux et la qualité de la réalisation. Elles ont été validées par les organismes officiels habilités.

De nouveaux produits apparaissent utilisant soit de la chènevotte, comme le Sativa Muris, bloc constructif composé de chènevotte et d'un éco-liant, soit de la fibre pour réaliser un paillage totalement écologique ${ }^{4}$.

Autre signe de la vitalité de la filière, plusieurs entreprises se sont crées depuis dix ans telles Agrochanvre, Agrofibres, Cavac Biomatériaux, etc. Elles viennent rejoindre les acteurs historiques (LCDA, Eurochanvre).

Les réalités actuelles de l'industrie chanvrière sont donc solides, appuyées sur des structures professionnelles éprouvées et une innovation prolifique. Ces réalités sont donc encourageantes.

\footnotetext{
1 Selon nos informations, cinq compagnies font cultiver 50000 ha pour alimenter leurs filatures.

2 À ce jour, on estime que 5000 tonnes de fibres sont ainsi utilisées en Europe ce qui représente plus de un million de $\mathrm{m}^{3}$ de laine de chanvre.

${ }^{3}$ http://www.construction-chanvre.asso.fr

${ }^{4}$ Sativa Muris et Protecsol ont été brevetés par Coopeval-Agrofibres.
} 


\section{Les perspectives sont extrêmement intéressantes et prometteuses !}

Elles s'appuient sur 3 facteurs complémentaires.

Le vert est à la mode! Cela a été souvent souligné et nous passerons donc rapidement. Mais il est vrai que l'écologie, le développement durable sont des valeurs montantes. Le Grenelle de l'Environnement a été un formidable marqueur qui a remis l'écologie et l'environnement au cœur des préoccupations de la société. Il s'agit bien d'un mouvement de fond dont profitent tous les produits végétaux. Le chanvre en particulier !

Le second facteur réside dans l'effort et les mobilisations de moyens matériels et intellectuels que sont les Pôles de Compétitivité.

Au premier rang, le pôle à vocation mondiale «Industrie et Agro Ressources » basé en Champagne Ardennes et Picardie où le chanvre occupe des programmes importants. Son voisin géographique, le Pôle Grand'Est est lui focalisé sur les fibres. Nul ne doute qu'avec l'implication d'instituts importants des progrès et des avancées significatives seront faites dans les années à venir. On peut aussi citer VEGETALIS et FINATHER 3.

Dans un autre registre, plusieurs pôles d'excellence rurale travaillent sur la production et la valorisation du chanvre industriel en Bretagne, Poitou- Charentes, Alsace-Lorraine, Isère ou Haute Saône. Toutes ces structures font avancer la connaissance et les technologies de production et de transformation du chanvre industriel.

Mais, à nos yeux, le facteur le plus important et le plus porteur d'avenir réside dans tous les travaux de recherche menées dans les Universités et Écoles d'Ingénieurs.

2010 a été riche en colloques et séminaires pour rendre compte et «publiciser » tous ces travaux, tant en France qu'à l'international.

En France, la journée de l'Université Bretagne Sud, en Juin, à Lorient, a focalisé sur les filières d'agro matériaux. À l'initiative du Pôle Fibres, à Metz, une autre rencontre faisait le point sur l'utilisation des fibres végétales dans l'industrie. Celle de l'ENSMA de Poitiers en Mars comportait une vingtaine de communications sur les propriétés des composants du chanvre. Certaines sont publiées dans la présente livraison.

J. Chamoin, F. Collet, S. Pretot et C. Lanos, dans leur travail sur le pouvoir absorbant de la chènevotte démontre qu'en raison de sa conductivité, le béton de chanvre et un «matériau accoustiquement et thermiquement intéressant » et ouvre des perspectives sur des traitements chimiques complémentaires. Avec l'aide du CSTB, S. Mom, S. Dartois, A. Ben Hamida, H. Dumontet et H. Boussa ont étudié le comportement thermique du béton de chanvre. Ces travaux serviront à caractériser le comportement effectif de ce matériau. Le CRITT Matériaux Poitou-Charentes de Rochefort est très en pointe sur les matériaux à base de chanvre. Il montre à partir de là que les bétons de chanvre constituent un matériau plus résistant et plus souple que les produits classiques. C. Bonnafous, F. Touchard, L. Chocinski-Arnault et C. Dever, s'intéressant au comportement mécanique de composites tissés à fibres de chanvre démontrent que les composites chanvre constituent une alternative à la problématique posée par le recyclage des fibres de verre. V. Placet, A. Bouali et P. Perré, ont étudié les tests de fatigue.

Tous ces travaux sont porteurs de développements industriels car plus on connaît la matière, mieux on peut l'utiliser.

Tous les ingrédients sont réunis pour que le chanvre industriel ait un avenir florissant. Un tel foisonnement d'initiatives, de recherches et d'investissements se traduira par des progrès substantiels!

Pour autant, l'ensemble des acteurs et les volumes traités ne constituent, pour le moment, qu'une petite activité. Pour aller plus loin, il faudra que la filière sécurise ses qualités et ses prix. Les utilisateurs veulent pouvoir compter sur des volumes certains, des qualités constantes et des prix stables. Pour aller plus loin, d'autres acteurs doivent se créer et apporter par leurs innovations, d'autres produits à base de chanvre et d'autres usages.

Le modèle économique idéal regroupe autour d'un même projet industriel des investisseurs agricoles, fournisseurs de chanvre, des hommes et des capitaux industriels qui transformeront et, idéalement, des utilisateurs. Ainsi constitué, le tour de table regroupe des acteurs qui ont tous intérêt au succès du projet. Les montages qui se développent actuellement sont basés, peu ou prou, sur ce mode de construction.

Notre souhait et notre espoir sont que les opportunités nées du mouvement porteur qu'est le développement durable, et que les travaux de nos jeunes chercheurs ici présentés se transforment en succès industriels durables.

Pierre Bouloc ${ }^{5}$

\footnotetext{
${ }^{5}$ Auteur de « Le Chanvre Industriel » Éditions France Agricole.
} 